\title{
GIẢI PHÁP LỤ๋A CHỌN PHƯƠNG PHÁP TRỘN ẢNH ĐỐI VỚI ẢNH VỆ TINH QUANG HỌC ĐỘ PHÂN GIẢI CAO (WorldView - 2)
}

\author{
ThS. ĐÕ TH! HOÀl \\ Viện Khoa học Đo đạc và Bản đồ
}

\section{Tóm tắt:}

Trộn ảnh là một công nghệ kết hợp các loại ảnh với nhau để tạo ra một loại ảnh mang nhiều thông tin hơn để tăng cường khả năng phân tích - nhận biết các đối tượng trên ảnh. Việc trộn ảnh vệ tinh quang học tức là kết hợp ảnh toàn sắc và ảnh đa phổ nhằm tạo ra ảnh vừa có độ phân giải cao về phổ (màu) như ảnh đa phổ lại vừa có độ phân giải cao về không gian như ảnh toàn sắc. Trong nội dung bài báo, các tác giả trình bày kết quả nghiên cứu thử nghiệm các phương pháp trộn dữ liệu ảnh vệ tinh độ phân giải cao (WorldView 2) và phân tích các chỉ số đánh giá chất lượng phổ trên ảnh sau khi trộn. Phương pháp trộn ảnh được nghiên cứu là: Phương pháp biến đổi hệ màu IHS cải tiến (Modified IHS); Phương pháp phân tích thành phần chính (PCA- Principal Component Analysis); Phương pháp nhân ảnh (Multiplicative); Phương pháp HPF (High Pass Filter); Phương pháp biến đổi Brovey.

\section{1. Đặt vấn đề}

Kỹ thuật viễn thám được sử dụng để nghiên cứu các đối tượng trên bề mặt Trái đất từ xa. Tuy nhiên, mỗi một loại tư liệu viễn thám chỉ có thể nghiên cứu một đối tượng hoặc một vài đặc tính của đối tượng trên bề mặt Trái đất phụ thuộc vào đặc điểm thu nhận tín hiệu của bộ cảm vệ tỉnh. Bộ cảm quang học cho phép phân loại lớp phủ dựa vào các đặc trưng phổ của các đối tượng trên ảnh. Trên thế giới và ở Việt Nam, đã có những nghiên cứu về khả năng trộn ảnh quang học [1], [2]. Các nghiên cứu đã thử nghiệm đối với dữ liệu ảnh Landsat, Spot $5 \ldots$ với các mức xử lý khác nhau. Sau khi trộn thì phương pháp đánh giá chất lượng của ảnh chủ yếu dựa trên phân tích bằng mắt. Do đó, trong nội dung bài báo, tác giả trình bày kết quả nghiên cứu thử nghiệm các chỉ số đánh giá chất lượng phổ của ảnh sau khi trộn và lựa chọn phương pháp trộn trên tư liệu vệ tinh quang học độ phân giải cao.

\section{Phương pháp trộn ảnh quang học}

\subsection{Phương pháp trộn ảnh}

Phương pháp trộn ảnh nhằm kết hợp dữ liệu của nhiều bộ cảm, nhiều thời điểm hoặc các thông tin khác nhau để tạo ra một ảnh mới mang nhiều thông tin hơn. Mục đích của việc trộn ảnh vệ tinh quang học là khai thác được nhiều thông tin nhằm tạo ra ảnh vừa có độ phân giải cao về phổ (màu) như ảnh đa phổ lại vừa có độ phân giải cao về không gian như ảnh toàn sắc. Trong nội dung bài báo, tác giả thử nghiệm các phương pháp trộn ảnh như phương pháp chuyển đổi hệ màu RGB-IHS, phương pháp chuyển đổi Brovey, phương pháp phân tích thành phần chính (Principal Component Analysis - PCA) và phương pháp nhân ảnh (Multiplicate).

a. Phương pháp chuyển đổi hệ màu $R G B-I H S$

Ngày chấp nhận đăng: 17/6/2016 
Ảnh số nói chung cũng như ảnh vệ tinh nói riêng được thể hiện bằng hệ màu $\mathrm{R}$ (đỏ) - G (xanh lá cây) - B (xanh lam). Khi hiển thị ảnh vệ tinh mỗi kênh ảnh được gán cho một màu nhất định. Hệ thống màu R-G-B được sử dụng rất rộng rãi trong đồ họa máy tính vì sự đơn giản và dễ sử dụng của nó. Tuy nhiên hệ thống màu R-G-B có nhược điểm là màu hiển thị sẽ phụ thuộc vào từng thành phần, hơn nữa hệ thống này không thể hiện được hết các sắc màu có thể có trong tự nhiên

Hệ thống màu HIS gồm ba thành phần: I (Intensity) cường độ màu, $\mathrm{H}$ (Hue) màu, $\mathrm{S}$ (Saturation) độ bão hòa màu. Ưu điểm của hệ thống này là thành phần cường độ màu độc lập với các thành phần còn lại và thể hiện nhiều màu trong tự nhiên hơn hệ thống RGB

- Các bước thực hiện như sau:

+ Ảnh tổ hợp màu gốc RGB chuyển sang hệ màu HIS;

+ Thay thế thành phần I - cường độ màu bằng kênh ảnh có độ phân giải cao; RGB.

+ Chuyển ngược tổ hợp HIS mới về

Theo [4] việc tính chuyển GRB - HIS GRB được thực hiện theo công thức sau:

$$
\left(\begin{array}{l}
I \\
v_{1} \\
v_{2}
\end{array}\right)=\left[\begin{array}{ccc}
\frac{1}{\sqrt{3}} & \frac{1}{\sqrt{3}} & \frac{1}{\sqrt{3}} \\
\frac{1}{\sqrt{6}} & \frac{1}{\sqrt{6}} & \frac{-2}{\sqrt{6}} \\
\frac{1}{\sqrt{2}} & \frac{-1}{\sqrt{2}} & 0
\end{array}\right]\left(\begin{array}{l}
R \\
G \\
B
\end{array}\right)
$$

Trong đó: ${ }_{1}, \psi_{2}$ là các giá trị trung gian Công thức chuyển đổi HIS thành RGB là:

$$
\left(\begin{array}{l}
R \\
G \\
B
\end{array}\right)=\left(\begin{array}{ccc}
\frac{1}{\sqrt{3}} & \frac{1}{\sqrt{6}} & \frac{1}{\sqrt{2}} \\
\frac{1}{\sqrt{3}} & \frac{1}{\sqrt{6}} & \frac{-1}{\sqrt{2}} \\
\frac{1}{\sqrt{3}} & \frac{-2}{\sqrt{6}} & 0
\end{array}\right)\left(\begin{array}{l}
I \\
v_{1} \\
v_{2}
\end{array}\right)
$$

Phương pháp chuyển đổi hệ màu IHS là một trong những phương pháp trộn ảnh cơ bản nhất, ban đầu được áp dụng để trộn ảnh đa phổ độ phân giải thấp với ảnh toàn sắc có độ phân giải cao, nhằm tăng cường độ phân giải và màu sắc của ảnh. Tuy nhiên, hiện nay, phương pháp chuyển đổi hệ màu IHS có thể áp dụng cho ảnh quang học kết hợp với ảnh SAR.

\section{b. Phương pháp phân tích thành phần} chính - PCA

Đây là một phương pháp sử dụng tương đối rộng rãi trong việc xử lý ảnh. Ảnh vệ tînh là lập dữ liệu đa kênh phổ điển hình có độ tương quan lớn hay có rất nhiều thông tin trùng lặp từ $\mathrm{PC} 1, \mathrm{PC} 2, \mathrm{PC} 3$...[4]. Vì vậy, sử dụng phương pháp này với mục đích:

- Phương pháp PCA được sử dụng để giảm số lượng các kênh phổ mà vẫn giữ được thông tin không bị thay đổi đáng kể;

- Dựa trên cơ sở các kênh phổ gần nhau có độ tương phản rất cao vì vậy các thông tin lặp lại rất lớn;

- Dựa trên các thông số thống kê.

+ Đầu tiên chuyển đổi ảnh đa phổ độ phân giải thấp (LRMIs) thành các thành phần chính không tương quan với nhau. Ảnh thành phần chính đầu tiên có chứa thông tin chung của tất cả các kênh ảnh sử dụng lúc nhập dữ liệu vào PCA, trong khi đó thông tin phổ là duy nhất với bất cứ kênh nào được ánh xạ tới các thành phần khác nhau.

+ Sau đó cũng giống như phương pháp $\mathrm{HIS}$, thành phần chính đầu tiên (PC1) đượC thay thế bằng ảnh toàn sắc độ phân giải cao (HRPIs).

+ Bước cuối cùng là tiến hành chuyển đổi PCA ngược trở lại để có được ảnh đa phổ độ phân giải cao ( HRMls - ảnh trộn).

Việc chuyển đổi thực hiện theo công thức sau: 


$$
\left[\begin{array}{c}
P C 1 \\
P C 2 \\
\ldots . . \\
P C n
\end{array}\right]=\left[\begin{array}{cccc}
v_{11} & v_{21} & \ldots . & v_{n 1} \\
v_{12} & v_{22} & \ldots . & v_{n 2} \\
\ldots . . & \ldots . . & \ldots . & \ldots . . \\
v_{1 n} & v_{2 n} & \ldots . & v_{n n}
\end{array}\right]\left[\begin{array}{c}
D N_{M S_{1}}^{l} \\
D N_{M S_{2}}^{l} \\
\ldots \ldots . . \\
D N_{M S_{n}}^{l}
\end{array}\right]
$$

Trong đó v là ma trận chuyển đổi:

$$
\begin{aligned}
& v=\left[\begin{array}{cccc}
v_{11} & v_{21} & \ldots . . & v_{1 n} \\
v_{21} & v_{22} & \ldots . . & v_{2 n} \\
\ldots . & \ldots . & \ldots . . & \ldots \ldots . \\
v_{1 n} & v_{2 n} & \ldots \ldots & v_{n n}
\end{array}\right] \\
& {\left[\begin{array}{c}
D N_{M S_{1}}^{h} \\
D N_{M S_{2}}^{h} \\
\ldots \ldots . \\
D N_{M S_{n}}^{h}
\end{array}\right]=\left[\begin{array}{cccc}
v_{11} & v_{21} & \ldots . & v_{n 1} \\
v_{12} & v_{22} & \ldots . & v_{n 2} \\
\ldots . . & \ldots . . & \ldots . & \ldots . . \\
v_{1 n} & v_{2 n} & \ldots . & v_{n n}
\end{array}\right]\left[\begin{array}{c}
P C_{1} \\
P C_{2} \\
\ldots \ldots . . \\
P C_{n}
\end{array}\right]}
\end{aligned}
$$

Trong đó: $P C 1, P C 2, P C n$ là thành phần chính trên các kênh ảnh $1,2, \ldots$.

$D N_{M S_{1}}^{l} \ldots . . D N_{M S_{n}}^{l}$ là giá trị độ xám ở ảnh đa phổ độ phân giải thấp kênh $1,2, \ldots$. .

$D N_{M S_{1}}^{h} \ldots . D N_{M S_{n}}^{h}$ là giá trị độ xám ở ảnh đa phổ độ phân giải cao kênh $1,2, \ldots$. .

\section{c. Phương pháp biến đổi Brovey}

Trong phương pháp trộn màu này các kênh ảnh mới được tạo ra thông qua việc cộng, trừ, nhân, chia các kênh ảnh màu với ảnh độ phân giải cao, sau đó tổ hợp các kênh mới lại để đạt được kết quả cuối cùng. Có rất nhiều công thức khác nhau được nhiều tác giả đề xuất. Một trong những phương pháp hiệu quả và phổ biến nhất là biến đổi của Brovey. Phương pháp biến đổi Brovey sử dụng thuật toán kết hợp màu sắc ảnh với dữ liệu phân giải cao được thể hiện theo công thức sau:

$D N_{\text {fived } S_{1}}=\frac{D N_{b_{1}}}{D N_{b_{1}}+D N_{b_{2}}+\ldots .+D N_{b_{n}}} D N_{P A N}$

Trong đó:
$D N_{D N_{\text {fruedusi }}}$ : Giá trị độ xám ở ảnh trộn

$D N_{b_{1}} \rightarrow D N_{b_{n}}$ : Giá trị độ xám ở ảnh đa phổ kênh $1,2, \ldots$ n

$D N_{P A N}$ : Giá trị độ xám ở ảnh toàn sắc

d. Phương pháp nhân ảnh (Multiplicative method)

Phương pháp nhân ảnh là phương pháp trộn ảnh kết hợp hai bộ dữ liệu bằng cách nhân từng pixel trong mỗi kênh ảnh $\mathrm{k}$ của dữ liệu đa phổ tương với pixel ảnh của dữ liệu toàn sắc. Để bù đắp cho sự ra tăng cường độ sáng, thực hiện căn bậc hai của tập dữ liệu kết quả. Theo [6] Công thức của phương pháp nhân ảnh như sau:

$$
F_{k(i, j)}=\sqrt{M S_{k(\mathrm{i}, \mathrm{j})} \times \operatorname{Pan}_{(i, j)}}
$$

Trong đó: $M S_{k(i, j)}$ là pixel tại hàng $\mathrm{i}$ cột $\mathrm{j}$ của kênh $\mathrm{k}$ trên ảnh đa phổ; $P a n_{(i, j)}$ là pixel tại hàng $\mathrm{i}$ cột $\mathrm{j}$ trên ảnh toàn sắc.

\section{e. Phương pháp trộn ảnh HPF}

Phương pháp trộn ảnh HPF lần đầu tiên được giới thiệu bởi Schowengerdt (1980) như một phương pháp để làm tăng độ phân giải không gian cho dữ liệu ảnh Landsat MSS. Phương pháp HPF về cơ bản bao gồm bổ sung của các chi tiết không gian được lấy từ ảnh toàn sắc độ phân giải cao PAN vào ảnh đa phổ độ phân giải thấp MS. Thông tin tần số cao được xác định bằng cách lọc thông tin trên ảnh toàn sắc với một bộ lọc High Pass, thông qua một điểm ảnh trung bình đơn giản tại một cửa sổ. Ví dụ, bộ lọc được sử dụng theo công thức (8).

$$
P_{H P F}=\frac{1}{9}\left[\begin{array}{ccc}
-1 & -1 & -1 \\
-1 & 8 & -1 \\
-1 & -1 & -1
\end{array}\right]
$$


Ở dạng đơn giản nhất, ma trận lọc High Pass được xác định bởi giá trị "-1" ở tất cả vị trí trừ vị trí trung tâm. High Pass là bộ lọc tính trung bình theo cửa sổ xung quanh mỗi điểm ảnh trên kênh toàn sắc. Các thành phần tần số cao sẽ được chồng lên hình ảnh toàn sắc. Ngoài ra, kết quả chia cho hai để bù đắp việc tăng giá trị độ sáng. Kỹ thuật này có thể cải thiện độ phân giải không gian cho dữ liệu ảnh tổ hợp và kênh ảnh riêng lẻ. Theo [6] công thức xác định theo (8):

$$
F_{k}=\frac{M S_{k}+P_{H P F}}{2}
$$

Trong đó: $M S_{k}$ : là Pixel của kênh trên ảnh đa phổ; $P_{H P F}$ Ảnh lọc tần số cao.

\subsection{Các chỉ số đánh giá chất lượng phổ trên ảnh sau khi trộn}

Chất lượng của ảnh sau khi trộn nhận được từ các phương pháp trình bày tại mục 2.1 sẽ được đánh giá dựa trên các thông tin về phổ trước và sau khi trộn. Phương pháp đánh giá chất lượng phổ trên ảnh sau khi trộn được phân tích thông qua các phương pháp: (1) So sánh chất lượng bằng mắt với ảnh trước khi trộn; (2) Phân tích vào histogram; (3) Phân tích các chỉ số đánh giá chất lượng phổ. Phương pháp đánh giá dựa vào việc so sánh chất lượng bằng mắt mang tính cảm quan, phụ thuộc vào kiến thức chuyên gia [8]. Phương pháp dựa vào phân tích histogram được sử dụng khi trộn ảnh đa phổ và ảnh toàn sắc do có chiều dài bước sóng có độ tương quan lớn giữa các kênh [9].

Do đó, các tác giả đề xuất phương pháp thử nghiệm đánh giá chất lượng ảnh sau khi trộn dựa vào các chỉ số đánh giá chất lượng phổ. Trong tài liệu nghiên cứu[3], [9], đã đề xuất sử dụng các chỉ số thống kê để đánh giá chất lượng phổ của ảnh sau khi trộn bao gồm chỉ số Bias, chỉ số chênh lệch Entropy (H), chỉ số ERGAS (Relative dimensionless global error in synthesis).
- Theo [3], chỉ Bias là độ lệch giữa giá trị trung bình của ảnh gốc và ảnh sau khi trộn. Chất lượng tốt nhất sẽ có giá trị gần bằng 0 :

$$
\text { Bias }=\frac{M S_{\text {mean }}-\text { fused }_{\text {mean }}}{M S_{\text {mean }}}=1-\frac{\text { fused }_{\text {mean }}}{M S_{\text {mean }}}
$$

Trong đó: $M S_{\text {mean }}$, fused mean là giá trị trung bình tương ứng của ảnh đa phổ và ảnh trộn và được tính theo công thức:

$$
\text { mean }=\frac{1}{m n} \sum_{i=j}^{m} \sum_{j=i}^{n} x_{\mathrm{ij}}
$$

- STD là độ lệch chuẩn tính trên mỗi kênh ảnh và được xác định theo công thức:

$$
S T D=\left[\frac{1}{n-1} \sum_{i=1}^{n}\left(M S_{i, j}-M S_{\text {mean }}\right)^{2}\right]^{1 / 2}
$$

- Theo [8], chỉ số chênh lệch Entropy: Sự khác biệt về entropy trên ảnh gốc và trên ảnh trộn

$$
H(x)=\sum_{i=1}^{n} p(i) \log _{2}\left(\frac{1}{p(i)}\right)=-\sum_{i=1}^{n} p(i) \log _{2} p(i)
$$

Trong đó: là xác suất xảy ra của giá trị i.

- Theo [8], chỉ số ERGAS (Relative dimensionless global error in synthesis) được đề xuất bởi Wald (2000) là một chỉ số đặc trưng cho chất lượng quá trình trộn ảnh dựa trên sai số trung bình chuẩn hóa của mỗi kênh trên ảnh sau khi trộn. Tăng chỉ số ERGAS đồng nghĩa với sự suy giảm hình ảnh trong quá trình trộn ảnh. Chỉ số ERGAS được tính như sau:

$$
\mathrm{ERGAS}=100 \frac{d h}{d l}\left[\frac{1}{n} \sum_{i=1}^{n}\left(\frac{R M S E^{2}}{\text { mean }^{2}}\right)\right]^{1 / 2}
$$

Trong đó: $d h / d l$ là tỷ số kích thước pixel của ảnh toàn sắc và ảnh đa phổ và $N$ là số lượng kênh ảnh.

RMSE là bình phương sai số được tính theo công thức sau:

$$
\operatorname{RMSE}\left(B_{i}\right)=\operatorname{bias}^{2}\left(B_{i}\right)+\operatorname{STD}^{2}\left(B_{i}\right)
$$

\section{Kết quả thử nghiệm}


Dữ liệu thử nghiệm đối với ảnh WorldView - 2. Đặc điểm của tư liệu thử nghiệm được thể hiện (xem bảng 1)

a. Phân tích kết quả trộn ảnh bằng quan sát trực tiếp

Kết quả thử nghiệm các phương pháp trộn ảnh được thể hiện: (xem hình 1)

(a) Ảnh gốc đa phổ trước khi trộn với tổ hợp màu (4:3:2);

(b) Ảnh gốc toàn sắc;

(c) Ảnh sau khi trộn theo phương pháp PCA

(d) Ảnh sau khi trộn theo phương pháp nhân ảnh;

(e) Ảnh sau khi trộn theo phương pháp IHS cải tiến;

(f) Ảnh sau khi trộn theo phương pháp Brovey;

(g) Ảnh sau khi trộn theo phương pháp HPF.

Tác giả so sánh đồ thị histogram của ảnh trước và sau khi trộn ảnh theo các phương pháp đã trình bày ở trên. (xem hình 2)

(a) Histogram của ảnh gốc đa phổ gốc;

(b) Histogram của ảnh sau khi trộn theo phương pháp PCA;

(c) Histogram của ảnh sau khi trộn theo phương pháp nhân ảnh;

(d) Histogram của ảnh sau khi trộn theo phương pháp Brovey;

(e) Histogram của ảnh sau khi trộn theo phương pháp IHS cải tiến;

(f) Ảnh sau khi trộn theo phương pháp HPF.

Trong (hình 2-d) ta thấy phương pháp Brovey sau khi trộn là rất nhỏ (xấp xỉ bằng $0)$. Do vậy khi phân tích Histogram không thể hiện đồ thị lên được.

c. Phân tích dựa trên các chỉ số đánh giá chất lượng phổ

Chất lượng phổ của các phương pháp trộn ảnh so với ảnh gốc được thể hiện qua bảng (xem bảng 2)

Qua kết quả thể hiện trong (hình 1), (hình 2) và (bảng 2), ta thấy phương pháp trộn ảnh theo phương pháp phương pháp IHS cải tiến cho kết quả tốt nhất với các chỉ số đánh giá chất lượng phổ tốt hơn so với các phương pháp khác. Phương pháp chuyển đổi hệ màu HIS cải tiến cho hình ảnh màu sắc tương tự như ảnh gốc. Đồ thị histogram của phương pháp chuyển đổi hệ màu HIS cải tiến có dạng tương quan với đồ thị Histogram của ảnh gốc. Đặc biệt chỉ số ERGAS của phương pháp chuyển đổi hệ màu HIS cải tiến là nhỏ nhất là 8.0397, chênh lệch về giá trị Bias là 0.6541 , chỉ số chênh lệch Entropy 0.1476 . Phương pháp nhân ảnh Multiplicative cho chất lượng phổ kém nhất với giá trị ERGAS là 25328.7

\section{Kết luận}

Phương pháp trộn dữ liệu ảnh đóng vai trò quan trọng trong các ứng dụng viễn thám, do có thể cung cấp nhiều thông tin hơn khi phân tích riêng từng dạng tư liệu. Qua kết quả phân tích quan sát trực tiếp và thông qua các chỉ số đánh giá chất lượng phổ trên ảnh sau khi trộn, tác giả lựa chọn phương pháp trộn ảnh theo phương pháp chuyển đổi hệ màu HIS cải tiến. Các chỉ số đánh giá chất lượng tương quan trên ảnh sau trộn ảnh cũng chỉ rõ phương pháp chuyển đổi hệ màu HIS cải tiến cho kết quả tốt nhất. $\bigcirc$

\section{Tài liệu tham khảo}

[1]. Chu Hải Tùng (2008), Nghiên cứu khả năng ứng dụng ảnh vệ tinh Radar và quang học để thành lập một số thông tin về lớp phủ mặt đất, Đề tài cấp Bộ, Bộ Tài nguyên và Môi trường.

[2]. Nguyen Van Trung, Jung-Hyun Choi, Joong-Sun Won. (2010). Fusion of ALOS PALSAR and ASTER data for landcover classification at Tonle Sap floodplain, Cambodia. Proc. SPIE Asia-Pacific Remote Sensing, Vol 7858.

[3]. Acerbi, F.W., Clevers, J.G.P.W., and Schaepman, M.E., 2006. The assessment 
Bảng 1: Tổng hợp dữ liệu thử nghiệm

\begin{tabular}{|c|l|}
\hline Giờ, ngày chụp & \multicolumn{1}{|c|}{ 010h38'/7/07/2010 } \\
\hline Mức độ phủ mây & $\sim 0 \%$ \\
\hline Điều kiện thời tiết & Nắng mạnh, tạo bóng đối tượng rõ \\
\hline Mức độ xử lý & $\begin{array}{l}\text { Tiền trực ảnh - OrthoReady } \\
\text { Standard, mức độ 2A đã được } \\
\text { hiệu chỉnh bức xạ }\end{array}$ \\
\hline Độ phân giải & $\begin{array}{l}\text { Kênh toàn sắc: 0.46m } \\
\text { Kênh đa phổ: 1.84m }\end{array}$ \\
\hline Khuôn dạng ảnh & GEOTIFF không nén, 11 Bit \\
\hline Hệ tọa độ tạm thời & "RPC" Geographic Lat/Lon -WGS84 \\
\hline $\begin{array}{c}\text { Độ lớn của cảnh } \\
\text { ảnh toàn sắc }\end{array}$ & $\begin{array}{l}\text { Số hàng: } 34418 \\
\text { Số cột: } 25960\end{array}$ \\
\hline $\begin{array}{c}\text { Độ lớn của cảnh } \\
\text { ảnh đa phổ }\end{array}$ & $\begin{array}{l}\text { Số hàng:8820 } \\
\text { Số cột: } 6295\end{array}$ \\
\hline
\end{tabular}

Bảng 2: Chỉ số đánh giá chất lượng phổ của các phương pháp trộn ảnh

\begin{tabular}{|c|c|c|c|}
\hline Phương pháp & Bias & $\mathrm{H}(\mathrm{x})$ & ERGAS \\
\hline PCA & 1.0127 & 0.0053 & 21.1194 \\
\hline Multiplicative & 341.408 & 0.0040 & 25328.7 \\
\hline Modif HIS & 0.6541 & 0.1476 & 8.0397 \\
\hline HPF & 0.3922 & 0.1970 & 9.2486 \\
\hline Brovey & 55.3270 & 0.0056 & 1153.3 \\
\hline
\end{tabular}

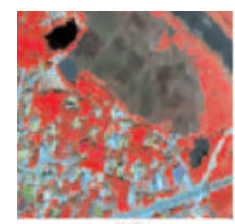

(a)

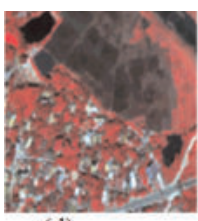

(d)

(e)

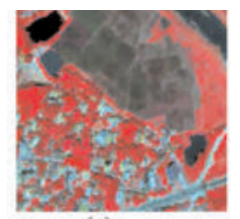

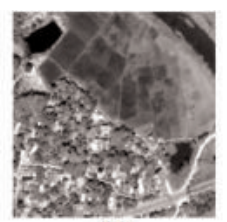

(b)

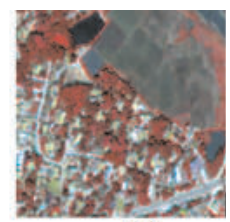

(c)

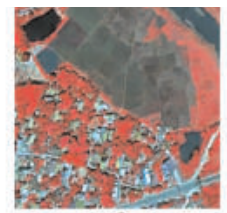

(f)

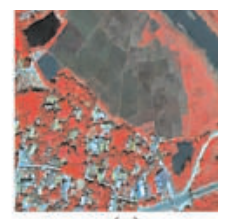

(g)

Hình 1: Kết quả các phương pháp trộn ảnh

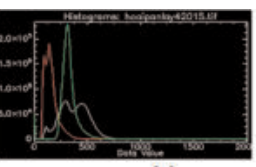

(a)

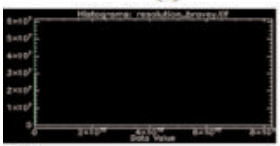

(d)

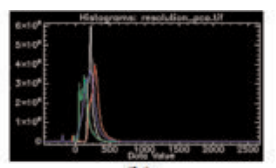

(b)

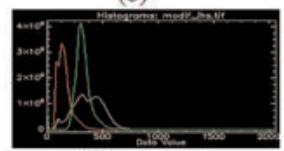

(e)

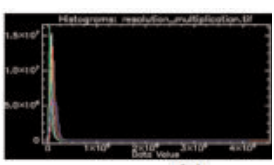

(c)

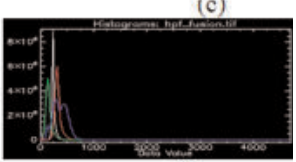

(f)

Hình 2: Phân tích đồ thị Histogram của các phương pháp trộn ảnh

of multi-sensor image fusion using wavelet transform for mapping the Brazalian Savana. International Journal of Applied Earth Observation and Geoinformation, 8, 278-288.

[4]. C. Pohl. C, (1999), Toll and methods for fusion of image of diferent spatial resolution, International Archives of photogrametry and remote Sensing, Vol.32, pat 7-4-3 W6, valadolid Spain 3-4 June.

[5]. C. Pohl \& J. L. Van Genderen (1998), Review article: Multisensor image fusion in remote sensing: concepts, methods and applications, International journal remote sensing, Vol. 19, No 5, pp.823-854.

[6]. Firouz Abdullah Al-Wassai, N.V. Kalyankar, Ali A. Al-Zuky (2011) Arithmetic and Frequency Filtering Methods of Pixel-Based Image Fusion Techniques. Journal-ref: International Journal of Advanced Research in Computer Science.

]7]. Yuhendra Yusuf, Josaphat Tetuko Sri Sumantyo, Hiroaki Kuze (2013), Spectral information analysis of image fusion data for remote sensing applications, Geocarto International, Vol. 28, No. 4, 291-310.

[8]. Shi, W., et al., 2005. Wavelet-based image fusion and quality assessment. International Journal of Applied Earth Observation and Geoinformation, 6, 241-251.

[9]. Yuhendra Yusuf, Josaphat Tetuko Sri Sumantyo, Hiroaki Kuze (2013), Spectral information analysis of image fusion data for remote sensing applications, Geocarto International, Vol. 28, No. 4, 291-310.0

(Xem tiếp trang 33) 